\title{
Efecto de la vía de administración de prostaglandinas sobre el porcentaje de gestación en becerras lecheras
}

\author{
Córdova-Izquierdo, A.'; Xolalpa-Campos, V.M.'; Ruiz-Lang, C.G.'; \\ Saltijeral-Oaxaca, J.A.'; Córdova-Jiménez, C.A. ${ }^{2}$
}

\begin{abstract}
${ }^{1}$ Departamento de Producción Agrícola y Animal, Universidad Autónoma Metropolitana, Unidad Xochimilco, Calzada del Hueso 1100, Colonia Villa Quietud, C.P. 04960, México, D.F. ${ }^{2}$ Facultad de Veterinaria, Universidad de León, España. E-mail: acordova@correo.xoc.uam.mx.
\end{abstract}

\begin{abstract}
Resumen
Córdova-Izquierdo, A.; Xolalpa-Campos, V.M.; Ruiz-Lang, C.G.; Saltijeral-Oaxaca, J.A.; Córdova-Jiménez, C.A.: Efecto de la vía de administración de prostaglandinas sobre el porcentaje de gestación en becerras lecheras. Rev. vet. 22: 1, 74-76, 2011. El objetivo del presente trabajo fue valorar el efecto de la vía de administración de prostaglandina (intramuscular e intravulvar) sobre el porcentaje de gestación en becerras lecheras. El estudio se llevó a cabo en una unidad de producción de bovinos lecheros ubicada en México, utilizando un diseño aleatorizado simple. Se seleccionaron 29 becerras Holstein Friesian con presencia de cuerpo lúteo a la palpación rectal. Los animales se dividieron en dos grupos. El grupo T1 (13 becerras) fue tratado el día 0 con una inyección intramuscular de $\mathrm{PGF}_{2} \alpha$ (Cloprostenol $530 \mathrm{mcg} / 2 \mathrm{ml}$ ) y al grupo T2 (16 becerras) se les administró el día 0 una inyección intravulvar de $\mathrm{PGF}_{2} \alpha$ (Dinoprost $10 \mathrm{mg} / 2 \mathrm{ml}$ ). El diagnóstico de gestación se realizó por palpación rectal. Los resultados fueron analizados estadísticamente por la prueba $X^{2}$ para comparación de tratamientos $(\mathrm{p}<0,05)$, con auxilio del paquete estadístico SAS. Los porcentajes de gestación fueron de 77 y $93 \%$ para T1 y T2 respectivamente, cifras que indican que los mejores resultados $(\mathrm{p}<0,05)$ se obtuvieron por vía intravulvar.
\end{abstract}

Palabras clave: vaquillas lecheras, sincronización de estros, prostaglandinas, inseminación a tiempo fijo, porcentaje de gestación.

\begin{abstract}
Córdova-Izquierdo, A.; Xolalpa-Campos, V.M.; Ruiz-Lang, C.G.; Saltijeral-Oaxaca, J.A.; Córdova-Jiménez, C.A.: Effect of route of administration of prostaglandins on the pregnancy rate in dairy heifers. Rev. vet. 22: 1, 74-76, 2011. The aim of this study was to assess the effect of route of administration of prostaglandins (intramuscular and intravulvar) on the pregnancy rate in dairy heifers. Work was carried out in a dairy farm located in Mexico, using a simple random design. A total of 29 Holstein Friesian heifers with presence of corpus luteum verified by rectal palpation, was selected. Animals were divided in two groups: (T1) with 13 calves which were administered on day 0 with an intramuscular injection of $\mathrm{PGF}_{2} \alpha$ (Cloprostenol $\left.530 \mathrm{mcg} / 2 \mathrm{ml}\right)$, and (T2) with 16 heifers, administered on day 0 with an intravulvar injection of $\mathrm{PGF}_{2} \alpha$ (Dinoprost $10 \mathrm{mg} / 2 \mathrm{ml}$ ). The pregnancy diagnosis was performed by rectal palpation. Results were statistically analyzed using $X^{2}$ test for comparison of treatments $(p<0.05)$ with the help of SAS software. The results were $77 \%$ and $93 \%$ of pregnancies for groups $\mathrm{T} 1$ and T2, respectively. It is concluded that the best results $(\mathrm{p}<0.05)$ were obtained with the intravulvar route.
\end{abstract}

Key words: dairy heifers, synchronization of estrus, prostaglandins, timed artificial insemination, pregnancy rate.

\section{INTRODUCCIÓN}

El estro puede ser inducido mediante la administración de $\mathrm{PGF}_{2} \alpha^{12}$; en la actualidad esta prostaglandina tiene gran importancia en reproducción animal debido a su poder luteolítico y al hecho que su uso haya sido

Recibido: 18 abril 2011 / Aceptado: 27 mayo 2011 aprobado en ganado lechero ${ }^{5}$. Las prostaglandinas son ácidos insaturados hidroxilados que poseen acción luteolítica, de tal manera que sus efectos son ejercidos cuando existe un cuerpo lúteo funcional en los ovarios ${ }^{6}$.

La utilización de prostaglandinas para la sincronización de estros es una invalorable herramienta ${ }^{2}$. También es útil durante el posparto temprano, para mejorar la involución uterina y la fertilidad en el ganado leche- 
ro ${ }^{8}$. En la vaca, como en otras especies de abasto, es esencial asegurar total fertilidad cuando se sincroniza el estro. La detección del estro en grupos de animales sincronizados puede ser difícil, sobre todo en unidades de producción extensivas, Tabla 1. Resultados obtenidos para ambos tratamientos (media aritmética).

\begin{tabular}{lccccc}
\hline tratamiento (n) & $\begin{array}{c}\text { vía de } \\
\text { administración }\end{array}$ & $\begin{array}{c}\text { producto } \\
\text { inyectado }\end{array}$ & $\begin{array}{c}\mathrm{N}^{\circ} \text { hembras } \\
\text { gestantes }\end{array}$ & $\begin{array}{c}\mathrm{N}^{\circ} \text { hembras } \\
\text { vacías }\end{array}$ & $\begin{array}{c}\% \text { de } \\
\text { gestación }\end{array}$ \\
\hline T1 (13) & intramuscular & Cloprostenol & 10 & 3 & $77 \%$ \\
T2 (16) & intravulvar & Dinoprost & 15 & 1 & $93 \%$ \\
total & - & - & 25 & 4 & - \\
\hline
\end{tabular}
porque muchos ejemplares pueden presentar calores al mismo tiempo ${ }^{1,9}$.

El objetivo del trabajo fue valorar el efecto de la vía de administración de $\mathrm{PGF}_{2} \alpha$ (intramuscular e intravulvar) sobre el porcentaje de gestación en becerras lecheras.

\section{MATERIAL Y MÉTODOS}

Lugar: el estudio se llevó a cabo en una unidad de producción de bovinos lecheros en el municipio de Ixtapaluca, al este del Estado de México, México. El clima de la región es templado subhúmedo, la temperatura presenta variaciones debido a que en el municipio hay zonas con mayor altura, siendo la media de $15.1^{\circ} \mathrm{C}$.

Animales: se seleccionaron 29 becerras Holstein Friesian, bajo condiciones de estabulación. Los criterios de selección fueron la presencia de cuerpo lúteo (CL) a la palpación rectal, condición corporal de 2,5 a 3 (escala 1-5) y edad promedio de 1,5 años, sometidas a manejo y alimentación convencionales. Para el estudio, las becerras se dividieron en dos grupos (TI y T2), aplicándoles prostaglandinas por dos vías, intramuscular (IM) e intravulvar (IV).

Tratamientos. Al grupo T1 (13 becerras) se les administró el día 0 una inyección intramuscular de $\mathrm{PGF}_{2} \alpha$ (Cloprostenol $530 \mathrm{mcg} / 2 \mathrm{ml}$ ). A las 96 horas del día 0 se efectuó la inseminación artificial (IA) a tiempo fijo y el diagnóstico de gestación se realizó por palpación rectal a los 45 días después de la IA. El grupo T2 (16 becerras) fue sometido a similares maniobras excepto la administración de $\mathrm{PGF}_{2} \alpha$ que se efectuó por inyección intravulvar (Dinoprost $10 \mathrm{mg} / 2 \mathrm{ml}$ ).

Estadísticas. Se efectuaron estadísticas descriptivas paramétricas de tendencia central y la prueba de $X^{2}$ para comparación de tratamientos $(\mathrm{p}<0,05)$, usando para ello el paquete estadístico JMP 8.1 de SAS ${ }^{10}$.

\section{RESULTADOS Y DISCUSIÓN}

En la Tabla 1 se puede observar que de un total de 13 becerras tratadas con Cloprostenol vía intramuscular (T1), 10 de ellas fueron positivas al diagnóstico de gestación y que de 16 becerras tratadas con Dinoprost vía intravulvar (T2), 15 resultaron positivas al diagnostico de gestación. En consecuencia, los porcentajes de gestación resultaron del 77 y 93\% en T1 y T2 respectivamente, diferencia que fue estadísticamente significativa $(\mathrm{p}<0,05)$.
La utilización de esquemas de sincronización de estro e IA a tiempo fijo en sistemas intensivos de producción lechera podría mejorar la eficiencia reproductiva ya que uno de los principales problemas que afectan a estos hatos, está asociado a la baja tasa de detección de estros ${ }^{11}$. En hatos con bajos rangos de detección de estro, los protocolos de sincronización proporcionan ventajas para la eficiencia reproductiva, mediante una adecuada detección de estros y aumento de las tasas de gestación ${ }^{3}$.

El protocolo utilizado en el presente trabajo generó altos porcentajes de gestación y redujo la posibilidad de error humano en la detección de estros. Se afirma que cuando se realiza IA a tiempo fijo, aumentan los porcentajes de gestación en vacas elegidas por detección de estro y presencia de cuerpo lúteo funcional ${ }^{3}$, pero esos resultados disminuyen cuando las vacas son inseminadas tras la detección de estros sin la verificación de la presencia del cuerpo lúteo. En cambio, otros indican que la sincronización de estro con $\mathrm{PGF}_{2} \alpha$ resulta exitosa si el ganado se insemina en un estro detectado debido a que se incrementa la tasa de detección de estro y el manejo de la IA resulta más eficiente comparado con la detección diaria del estro sin ningún programa de sincronización ${ }^{4}$. Además, cuando las vacas reciben una IA a tiempo fijo y detección de estro, la tasa de preñez es de aproximadamente la mitad de las vacas servidas con celo detectado.

Los porcentajes de gestación aquí obtenidos indican que la mejor vía de administración de prostaglandina fue la intravulvar. En otras investigaciones se encontró que la vía intramuscular resultó práctica en $\mathrm{su}$ aplicación, pero su resultado no fue muy efectivo en la respuesta al estro y porcentaje de gestaciones tras la IA a tiempo fijo ${ }^{7}$.

En conclusión, el tipo usado de prostaglandina ( $D i$ noprost) por vía intravulvar, mejoró el porcentaje de gestación en comparación al obtenido con Cloprostenol por vía intramuscular. No obstante, ambos esquemas de sincronización de estro e IA a tiempo fijo constituyen maniobras prácticas capaces de mejorar la eficiencia reproductiva, ya que uno de los principales problemas que afectan a los hatos lecheros de producción intensiva, está asociado a la baja tasa de detección de estros y en consecuencia, a la disminución del porcentaje de gestaciones obtenidas.

\section{REFERENCIAS}

1. Bartolome J, Sozzi A, McHale J, Melendez P, Arteche AC, Silvestre FT, Kelbert D, Swift K, Archbald LF, Thatcher WW. 2005. Resynchronization of ovulation and 
timed insemination in lactating dairy cows I: use of the Ovsynch and Heatsynch protocols after non-pregnancy diagnosis by ultrasonography. Theriogenology 63: 16171627.

2. Cavestany D. 2004. Resultados experimentales en lechería, Ed. Instituto Nacional de Investigación Agropecuaria, Montevideo, $20 \mathrm{p}$.

3. Cerri RL, Santos JE, Juchem SO, Galvão KN, Chebel RC. 2004. Timed artificial insemination with estradiol cypionate or insemination at estrus in high-producing dairy cows. J Dairy Sci 87: 3704-3715.

4. Fricke MP. 2001. Manipulación de la función ovárica. Novedades lácteas, reproducción y selección genética (Instituto Babcock, Universidad de Wisconsin), 605: 1-14.

5. Gumen A, Seguin B. 2003. Ovulation rate alter GnRH or $\mathrm{PGF}_{2 \propto}$ administration in early postpartum dairy cows. Theriogenology 60: 341-348.

6. Hirsbrunner G. 2002. Effect of prostaglandin E2, DLcloprostenol, and prostaglandin E2 in combination with D-cloprostenol on uterine motility during diestrus in experimental cows. Anim Reprod Sci 79: 17-32.
7. Martineau R. 2003. Dinoprost versus Cloprostenol: does route of injection modulate their efficacy in dairy cattle. The Bov Pract 37: 10-19.

8. Melendez P, McHale J, Bartolome J, Archbald LF, Donovan GA. 2004. Uterine involution and fertility of Holstein cows subsequent to early postpartum PGF2 $\alpha$ treatment for acute puerperal metritis. J Dairy Sci 87 : 3238-3246.

9. Peters M, Pursley J. 2002. Fertility of lactating dairy cows treated whit Ovsynh after presyncronization injections of PGF $2 \propto$ and GnRH. J Dairy Sci 85: 2403-2405.

10. SAS. 1995. Statistics and graphic guide, JMP 8.1, ed. SAS Institute, Toronto.

11. Sepúlveda N, Risopatrón J, Rodríguez F, Rodero E. 2003. Fertilidad en vacas lecheras asociada a la sincronización de celos e inseminación a tiempo fijo utilizando GnRh y PGF2 $\propto$. Revista Cientifica FCV (Universidad de Zulia, Venezuela) 13: 182-186.

12. Xiang-Dong Z. 2003. Reproduction in female yaks (Bos grunniens) and opportunities for improvement. Theriogenology 59: 1303-1321. 